\title{
Existence of Almost Periodic Solutions to Nth-Order Neutral Differential Equations with Piecewise Constant Arguments
}

\author{
Rong-Kun Zhuang \\ Department of Mathematics, Huizhou University, Huizhou 516007, China \\ Correspondence should be addressed to Rong-Kun Zhuang, zhuangrk@hzu.edu.cn \\ Received 12 November 2011; Accepted 12 December 2011 \\ Academic Editor: Simeon Reich
}

Copyright (C) 2012 Rong-Kun Zhuang. This is an open access article distributed under the Creative Commons Attribution License, which permits unrestricted use, distribution, and reproduction in any medium, provided the original work is properly cited.

We present some conditions for the existence and uniqueness of almost periodic solutions of $N$ th-order neutral differential equations with piecewise constant arguments of the form $(x(t)+$ $p x(t-1))^{(N)}=q x([t])+f(t)$, here $[\cdot]$ is the greatest integer function, $p$ and $q$ are nonzero constants, $N$ is a positive integer, and $f(t)$ is almost periodic.

\section{Introduction}

In this paper we study certain functional differential equations of neutral delay type with piecewise constant arguments of the form

$$
(x(t)+p x(t-1))^{(N)}=q x([t])+f(t),
$$

here $[\cdot]$ is the greatest integer function, $p$ and $q$ are nonzero constants, $N$ is a positive integer, and $f(t)$ is almost periodic. Throughout this paper, we use the following notations: $\mathbb{R}$ is the set of reals; $\mathbb{R}^{+}$the set of positive reals; $\mathbb{Z}$ the set of integers; that is, $\mathbb{Z}=\{0, \pm 1, \pm 2, \ldots\} ; \mathbb{Z}^{+}$the set of positive integers; $\mathbb{C}$ denotes the set of complex numbers. A function $x: \mathbb{R} \rightarrow \mathbb{R}$ is called a solution of (1.1) if the following conditions are satisfied:

(i) $x$ is continuous on $\mathbb{R}$;

(ii) the $N$ th-order derivative of $x(t)+p(t) x(t-1)$ exists on $\mathbb{R}$ except possibly at the points $t=n, n \in \mathbb{Z}$, where one-sided $N$ th-order derivatives of $x(t)+p(t) x(t-1)$ exist;

(iii) $x$ satisfies (1.1) on each interval $(n, n+1)$ with integer $n \in \mathbb{Z}$. 
Differential equations with piecewise constant arguments are usually referred to as a hybrid system, and could model certain harmonic oscillators with almost periodic forcing. For some excellent works in this field we refer the reader to [1-5] and references therein, and for a survey of work on differential equations with piecewise constant arguments we refer the reader to [6].

In paper [1, 2], Yuan and $\mathrm{Li}$ and $\mathrm{He}$, respectively, studied the existence of almost periodic solutions for second-order equations involving the argument $2[(t+1) / 2]$ in the unknown function. In paper [3], Seifert intensively studied the special case of (1.1) for $N=2$ and $|p|<1$ by using different methods. However, to the best of our knowledge, there are no results regarding the existence of almost periodic solutions for $N$ th-order neutral differential equations with piecewise constant arguments as (1.1) up to now.

Motivated by the ideas of Yuan [1] and Seifert [3], in this paper we will investigate the existence of almost periodic solutions to (1.1). Both the cases when $|p|<1$ and $|p|>1$ are considered.

\section{The Main Results}

We begin with some definitions, which can be found (or simply deduced from the theory) in any book, say [7], on almost periodic functions.

Definition 2.1. A set $K \subset \mathbb{R}$ is said to be relatively dense if there exists $L>0$ such that $[a, a+$ $L] \cap K \neq \emptyset$ for all $a \in \mathbb{R}$.

Definition 2.2. A bounded continuous function $f: \mathbb{R} \rightarrow \mathbb{R}$ (resp., $\mathbb{C}$ ) is said to be almost periodic if the $\varepsilon$-translation set of $f$

$$
T(f, \varepsilon)=\{\tau \in \mathbb{R}:|f(t+\tau)-f(t)|<\varepsilon \forall t \in \mathbb{R}\}
$$

is relatively dense for each $\varepsilon>0$. We denote the set of all such function $f$ by $\operatorname{AP}(\mathbb{R}, \mathbb{R})$ (resp., $\operatorname{AP}(\mathbb{R}, \mathbb{C})$ ).

Definition 2.3. A sequence $x: \mathbb{Z} \rightarrow \mathbb{R}^{k}$ (resp., $\mathbb{C}^{k}$ ), $k \in \mathbb{Z}, k>0$, denoted by $\left\{x_{n}\right\}$, is called an almost periodic sequence if the $\varepsilon$-translation set of $\left\{x_{n}\right\}$

$$
T\left(\left\{x_{n}\right\}, \varepsilon\right)=\left\{\tau \in \mathbb{Z}:\left|x_{n+\tau}-x_{n}\right|<\varepsilon \forall n \in \mathbb{Z}\right\}
$$

is relatively dense for each $\varepsilon>0$, here $|\cdot|$ is any convenient norm in $\mathbb{R}^{k}\left(\right.$ resp., $\left.\mathbb{C}^{k}\right)$. We denote the set of all such sequences $\left\{x_{n}\right\}$ by $\operatorname{APS}\left(\mathbb{Z}, \mathbb{R}^{k}\right)$ (resp., $\operatorname{APS}\left(\mathbb{Z}, \mathbb{C}^{k}\right)$ ).

Proposition 2.4. $\left\{x_{n}\right\}=\left\{\left(x_{n 1}, x_{n 2}, \ldots, x_{n k}\right)\right\} \in \operatorname{APS}\left(\mathbb{Z}, \mathbb{R}^{k}\right)\left(\right.$ resp., $\left.\operatorname{APS}\left(\mathbb{Z}, \mathbb{C}^{k}\right)\right)$ if and only if $\left\{x_{n i}\right\} \in \operatorname{APS}(\mathbb{Z}, \mathbb{R}) \quad($ resp., $\operatorname{APS}(\mathbb{Z}, \mathbb{C})), i=1,2, \ldots, k$.

Proposition 2.5. Suppose that $\left\{x_{n}\right\} \in \operatorname{APS}(\mathbb{Z}, \mathbb{R}), f \in \operatorname{AP}(\mathbb{R}, \mathbb{R})$. Then the sets $T(f, \varepsilon) \cap \mathbb{Z}$ and $T\left(\left\{x_{n}\right\}, \varepsilon\right) \cap T(f, \varepsilon)$ are relatively dense. 
Now one rewrites (1.1) as the following equivalent system

$$
\begin{array}{cc}
(x(t)+p x(t-1))^{\prime}=y_{1}(t), & \left(2.3_{1}\right) \\
y_{1}^{\prime}(t)=y_{2}(t), & \left(2.3_{2}\right) \\
\vdots & \vdots \\
y_{N-2}^{\prime}(t)=y_{N-1}(t), & \left(2.3_{N-1}\right) \\
y_{N-1}^{\prime}(t)=q x([t])+f(t) . & \left(2.3_{N}\right)
\end{array}
$$

Let $\left(x(t), y_{1}(t), \ldots, y_{N-1}(t)\right)$ be solutions of system (2.3) on $\mathbb{R}$, for $n \leq t<n+1, n \in \mathbb{Z}$, using $\left(2.3_{N}\right)$ we obtain

$$
y_{N-1}(t)=y_{N-1}(n)+q x(n)(t-n)+\int_{n}^{t} f\left(t_{1}\right) d t_{1}
$$

and using this with $\left(2.3_{N-1}\right)$ we obtain

$$
y_{N-2}(t)=y_{N-2}(n)+y_{N-1}(n)(t-n)+\frac{1}{2} q x(n)(t-n)^{2}+\int_{n}^{t} \int_{n}^{t_{2}} f\left(t_{1}\right) d t_{1} d t_{2} .
$$

Continuing this way, and, at last, we get

$$
\begin{aligned}
x(t)+p x(t-1)= & x(n)+p x(n-1)+y_{1}(n)(t-n)+\frac{1}{2} y_{2}(n)(t-n)^{2}+\cdots \\
& +\frac{1}{(N-1) !} y_{N-1}(n)(t-n)^{N-1}+\frac{1}{N !} q x(n)(t-n)^{N} \\
& +\int_{n}^{t} \int_{n}^{t_{N}} \cdots \int_{n}^{t_{2}} f\left(t_{1}\right) d t_{1} d t_{2} \cdots d t_{N} .
\end{aligned}
$$

Since $x(t)$ must be continuous at $n+1$, using these equations we get for $n \in \mathbb{Z}$,

$$
\begin{aligned}
x(n+1)= & \left(1-p+\frac{q}{N !}\right) x(n)+y_{1}(n)+\frac{1}{2 !} y_{2}(n)+\cdots+\frac{1}{(N-1) !} y_{N-1}(n)+p x(n-1) \\
& +f_{n}^{(1)}, \\
y_{1}(n+1)= & \frac{q}{(N-1) !} x(n)+y_{1}(n)+y_{2}(n)+\frac{1}{2 !} y_{3}(n)+\cdots+\frac{1}{(N-2) !} y_{N-1}(n)+f_{n}^{(2)},
\end{aligned}
$$

$y_{N-2}(n+1)=\frac{q}{2} x(n)+y_{N-2}(n)+y_{N-1}(n)+f_{n}^{(N-1)}$,

$y_{N-1}(n+1)=q x(n)+y_{N-1}(n)+f_{n}^{(N)}$, 
where

$$
\begin{gathered}
f_{n}^{(1)}=\int_{n}^{n+1} \int_{n}^{t_{N}} \cdots \int_{n}^{t_{2}} f\left(t_{1}\right) d t_{1} d t_{2} \cdots d t_{N}, \ldots, f_{n}^{(N-1)}=\int_{n}^{n+1} \int_{n}^{t_{2}} f\left(t_{1}\right) d t_{1} d t_{2} \\
f_{n}^{(N)}=\int_{n}^{n+1} f\left(t_{1}\right) d t_{1} .
\end{gathered}
$$

Lemma 2.6. If $f \in A P(\mathbb{R}, \mathbb{R})$, then sequences $\left\{f_{n}^{(i)}\right\} \in A P S(\mathbb{Z}, \mathbb{R}), i=1,2, \ldots, N$.

Proof. We typically consider $\left\{f_{n}^{(1)}\right\}$ for all $\varepsilon>0$ and $\tau \in T(f, \varepsilon) \cap \mathbb{Z}$, we have

$$
\begin{aligned}
\left|f_{n+\tau}^{(1)}-f_{n}^{(1)}\right| & =\left|\int_{n+\tau}^{n+\tau+1} \int_{n+\tau}^{t_{N}} \cdots \int_{n+\tau}^{t_{2}} f\left(t_{1}\right) d t_{1} d t_{2} \cdots d t_{N}-\int_{n}^{n+1} \int_{n}^{t_{N}} \cdots \int_{n}^{t_{2}} f\left(t_{1}\right) d t_{1} d t_{2} \cdots d t_{N}\right| \\
& \leq \int_{n}^{n+1} \int_{n}^{t_{N}} \cdots \int_{n}^{t_{2}}\left|f\left(t_{1}+\tau\right)-f\left(t_{1}\right)\right| d t_{1} d t_{2} \cdots d t_{N} \\
& \leq \frac{\varepsilon}{N !}
\end{aligned}
$$

From Definition 2.3, it follows that $\left\{f_{n}^{(1)}\right\}$ is an almost periodic sequence. In a manner similar to the proof just completed, we know that $\left\{f_{n}^{(2)}\right\},\left\{f_{n}^{(3)}\right\}, \ldots,\left\{f_{n}^{(N)}\right\}$ are also almost periodic sequences. This completes the proof of the lemma.

Lemma 2.7. The system of difference equations

$$
\begin{aligned}
c_{n+1} & =\left(1-p+\frac{q}{N !}\right) c_{n}+d_{n}^{(1)}+\frac{1}{2 !} d_{n}^{(2)}+\cdots+\frac{1}{(N-1) !} d_{n}^{(N-1)}+p c_{n-1}+f_{n}^{(1)}, \\
d_{n+1}^{(1)} & =\frac{q}{(N-1) !} c_{n}+d_{n}^{(1)}+d_{n}^{(2)}+\frac{1}{2 !} d_{n}^{(3)}+\cdots+\frac{1}{(N-2) !} d_{n}^{(N-1)}+f_{n}^{(2)}, \\
& \vdots \\
d_{n+1}^{(N-2)} & =\frac{q}{2} c_{n}+d_{n}^{(N-2)}+d_{n}^{(N-1)}+f_{n}^{(N-1)}, \\
d_{n+1}^{(N-1)} & =q c_{n}+d_{n}^{(N-1)}+f_{n}^{(N)},
\end{aligned}
$$

has solutions on $\mathbb{Z}$; these are in fact uniquely determined by $c_{0}, c_{-1}, d_{0}^{(1)}, \ldots, d_{0}^{(N-1)}$.

Proof. It is easy to check that $c_{n}, d_{n}^{(i)}, i=1,2, \ldots, N-1$ are uniquely determined in term of $c_{0}, c_{-1}, d_{0}^{(1)}, d_{0}^{(2)}, \ldots, d_{0}^{(N-1)}$ for $n \in \mathbb{Z}^{+}$. For $n=-1,\left(2.10_{N}\right)$ uniquely determines $d_{-1}^{(N-1)}$, $\left(2.10_{N-1}\right)$ uniquely determines $d_{-1}^{(N-2)}, \ldots,\left(2.10_{2}\right)$ uniquely determines $d_{-1}^{(1)}$, and thus since $p \neq 0,\left(2.10_{1}\right)$ uniquely determines $c_{-2}$. So $c_{-1}, c_{-2}, d_{-1}^{(1)}, d_{-1}^{(2)}, \ldots, d_{-1}^{(N-1)}$ are determined. Continuing in this way, we establish the lemma. 
Lemma 2.8. For any solution $\left(c_{n}, d_{n}^{(1)}, d_{n}^{(2)}, \ldots, d_{n}^{(N-1)}\right), n \in \mathbb{Z}$, of system (2.10), there exists a solution $\left(x(t), y_{1}(t), y_{2}(t), \ldots, y_{N-1}(t)\right), t \in R$, of $(2.3)$ such that $x(n)=c_{n}, y_{1}(n)=d_{n}^{(1)}, \ldots$, $y_{N-1}(n)=d_{n}^{(N-1)}, n \in \mathbb{Z}$.

Proof. Define

$$
\begin{aligned}
w(t)= & c_{n}+p c_{n-1}+d_{n}^{(1)}(t-n)+\frac{1}{2 !} d_{n}^{(2)}(t-n)^{2}+\cdots \\
& +\frac{1}{(N-1) !} d_{n}^{(N-1)}(t-n)^{N-1}+\frac{1}{N !} q c_{n}(t-n)^{N}+\int_{n}^{t} \int_{n}^{t_{N}} \cdots \int_{n}^{t_{2}} f\left(t_{1}\right) d t_{1} d t_{1} \cdots d t_{N},
\end{aligned}
$$

for $n \leq t<n+1, n \in \mathbb{Z}$. It can easily be verified that $w(t)$ is continuous on $\mathbb{R}$; we omit the details.

Define $x(t)=\varphi(t),-1 \leq t \leq 0$, where $\varphi(t)$ is continuous, and $\varphi(0)=c_{0}, \varphi(-1)=c_{-1}$;

$$
\begin{array}{ll}
x(t)=\frac{[w(t+1)-\varphi(t+1)]}{p}, & -2 \leq t<-1, \\
x(t)=\frac{[w(t+1)-x(t+1)]}{p}, & -3 \leq t<-2 .
\end{array}
$$

Continuing this way, we can define $x(t)$ for $t<0$. Similarly, define

$$
\begin{array}{ll}
x(t)=-p \varphi(t-1)+w(t), & 0 \leq t<1, \\
x(t)=-p x(t-1)+w(t), & 1 \leq t<2,
\end{array}
$$

continuing in this way $x(t)$ is defined for $t \geq 0$, and so $x(t)$ is defined for all $t \in \mathbb{R}$.

Next, define $y_{1}(t)=w^{\prime}(t), y_{2}(t)=w^{\prime \prime}(t), \ldots, y_{N-1}(t)=w^{(N-1)}(t), t \neq n \in \mathbb{Z}$, and by the appropriate one-sided derivative of $w^{\prime}(t), w^{\prime \prime}(t), \ldots, w^{(N-1)}(t)$ at $n \in \mathbb{Z}$. It is easy to see that $y_{1}(t), y_{2}(t), \ldots, y_{N-1}(t)$ are continuous on $\mathbb{R}$, and $\left(x(n), y_{1}(n), y_{2}(n), \ldots, y_{N-1}(n)\right)=$ $\left(c_{n}, d_{n}^{(1)}, d_{n}^{(2)}, \ldots, d_{n}^{(N-1)}\right)$ for $n \in \mathbb{Z}$; we omit the details.

Next we express system (2.7) in terms of an equivalent system in $\mathbb{R}^{N+1}$ give by

$$
v_{n+1}=A v_{n}+h_{n}
$$


where

$$
\begin{gathered}
A=\left(\begin{array}{cccccc}
1-p+\frac{q}{N !} & 1 & \frac{1}{2 !} & \cdots & \frac{1}{(N-1) !} & p \\
\frac{q}{(N-1) !} & 1 & 1 & \cdots & \frac{1}{(N-2) !} & 0 \\
\cdot & \cdot & . & . & \cdot & \cdot \\
\frac{q}{2 !} & 0 & 0 & \cdots & 1 & 0 \\
q & 0 & 0 & \cdots & 1 & 0 \\
1 & 0 & 0 & \cdots & 0 & 0
\end{array}\right), \\
v_{n}=\left(x(n), y_{1}(n), y_{2}(n), \ldots, y_{N-1}, x(n-1)\right)^{T}, \\
h_{n}=\left(f_{n}^{(1)}, f_{n}^{(2)}, \ldots, f_{n}^{(N)}, 0\right)^{T} .
\end{gathered}
$$

Lemma 2.9. Suppose that all eigenvalues of $A$ are simple (denoted by $\lambda_{1}, \lambda_{2}, \ldots, \lambda_{N+1}$ ) and $\left|\lambda_{i}\right| \neq 1$, $1 \leq i \leq N+1$. Then system (2.14) has a unique almost periodic solution.

Proof. From our hypotheses, there exists a $(N+1) \times(N+1)$ nonsingular matrix $P$ such that $P A P^{-1}=\Lambda$, where $\Lambda=\operatorname{diag}\left(\lambda_{1}, \lambda_{2}, \ldots, \lambda_{N+1}\right)$ and $\lambda_{1}, \lambda_{2}, \ldots, \lambda_{N+1}$ are the distinct eigenvalues of $A$. Define $\bar{v}_{n}=P v_{n}$, then (2.14) becomes

$$
\bar{v}_{n+1}=\Lambda \bar{v}_{n}+\bar{h}_{n}
$$

where $\bar{h}_{n}=P h_{n}$.

For the sake of simplicity, we consider first the case $\left|\lambda_{1}\right|<1$. Define

$$
\bar{v}_{n 1}=\sum_{m \leq n} \lambda_{1}^{n-m} \bar{h}_{(m-1) 1}
$$

where $\bar{h}_{n}=\left(\bar{h}_{n 1}, \bar{h}_{n 2}, \ldots, \bar{h}_{n(N+1)}\right)^{T}, n \in \mathbb{Z}$. Clearly $\left\{\bar{h}_{n 1}\right\}$ is almost periodic, since $\bar{h}_{n}=P h_{n}$ and $\left\{h_{n}\right\}$ is. For $\tau \in T\left(\left\{\bar{h}_{n 1}\right\}, \varepsilon\right)$, we have

$$
\begin{aligned}
& \left|\bar{v}_{(n+\tau) 1}-\bar{v}_{n 1}\right|=\left|\sum_{m \leq n+\tau} \lambda_{1}^{n+\tau-m} \bar{h}_{(m-1) 1}-\sum_{m \leq n} \lambda_{1}^{n-m} \bar{h}_{(m-1) 1}\right| \\
& \text { (letting } m=m^{\prime}+\tau \text {, then replacing } m^{\prime} \text { by } m \text { ) } \\
& =\left|\sum_{m \leq n} \lambda_{1}^{n-m} \bar{h}_{(m+\tau-1) 1}-\sum_{m \leq n} \lambda_{1}^{n-m} \bar{h}_{(m-1) 1}\right| \\
& =\left|\sum_{m \leq n} \lambda_{1}^{n-m}\left(\bar{h}_{(m+\tau-1) 1}-\bar{h}_{(m-1) 1}\right)\right| \\
& \leq \frac{\varepsilon}{1-\left|\lambda_{1}\right|} \text {, }
\end{aligned}
$$

this shows that $\left\{\bar{v}_{n 1}\right\} \in \operatorname{APS}(\mathbb{Z}, \mathbb{C})$. 
If $\left|\lambda_{i}\right|<1,2 \leq i \leq N+1$, in a manner similar to the proof just completed for $\lambda_{1}$, we know that $\left\{\bar{v}_{n i}\right\} \in P A S(\mathbb{Z}, \mathbb{C}), 2 \leq i \leq N+1$, and so $\left\{\bar{v}_{n}\right\} \in \operatorname{APS}\left(\mathbb{Z}, \mathbb{C}^{N+1}\right)$. It follows easily that then $\left\{P^{-1} \bar{v}_{n}\right\}=\left\{v_{n}\right\} \in \operatorname{APS}\left(\mathbb{Z}, \mathbb{R}^{N+1}\right)$ and our lemma follows.

Assume now $\left|\lambda_{1}\right|>1$. Now define

$$
\bar{v}_{n 1}=\sum_{m \leq n} \lambda_{1}^{m-n} \bar{h}_{(m-1) 1}, \quad n \in \mathbb{Z} .
$$

As before, the fact that $\left\{\bar{v}_{n 1}\right\} \in \operatorname{APS}(\mathbb{Z}, \mathbb{C})$ follows easily from the fact that $\left\{\bar{h}_{n 1}\right\} \in \operatorname{APS}(\mathbb{Z}, \mathbb{C})$. So in every possible case, we see that each component $v_{n i}, i=1,2, \ldots, N+1$, of $v_{n}$ is almost periodic and so $\left\{v_{n}\right\} \in \operatorname{APS}\left(\mathbb{Z}, \mathbb{R}^{N+1}\right)$.

The uniqueness of this almost periodic solution $\left\{v_{n}\right\}$ of (2.14) follows from the uniqueness of the solution $\bar{v}_{n}$ of (2.16) since $P^{-1} \bar{v}_{n}=v_{n}$, and the uniqueness of $\bar{v}_{n}$ of (2.16) follows, since if $\widetilde{v}_{n}$ were a solution of (2.16) distinct from $\bar{v}_{n}, u_{n}=\bar{v}_{n}-\widetilde{v}_{n}$ would also be almost periodic and solve $u_{n+1}=\Lambda u_{n}, n \in \mathbb{Z}$. But by our condition on $\Lambda$, it follows that each component of $u_{n}$ must become unbounded either as $n \rightarrow \infty$ or as $n \rightarrow-\infty$, and that is impossible, since it must be almost periodic. This proves the lemma.

Lemma 2.10. Suppose that conditions of Lemma 2.9 hold, $w(t)$ is as defined in the proof of Lemma 2.8 with $\left(c_{n}, d_{n}^{(1)}, d_{n}^{(2)}, \ldots, d_{n}^{(N-1)}\right)$ the unique first $N$ components of the almost periodic solution of (2.14) given by Lemma 2.9, then $w(t)$ is almost periodic.

Proof. For $\tau \in T\left(\left\{c_{n}\right\}, \varepsilon\right) \cap T\left(\left\{d_{n}^{(1)}\right\}, \varepsilon\right) \cap T\left(\left\{d_{n}^{(2)}\right\}, \varepsilon\right) \cap \cdots \cap T\left(\left\{d_{n}^{(N-1)}\right\}, \varepsilon\right) \cap T(f, \varepsilon)$,

$$
\begin{aligned}
& |w(t+\tau)-w(t)| \\
& =\mid\left(c_{n+\tau}-c_{n}\right)+p\left(c_{n+\tau-1}-c_{n-1}\right)+\left(d_{n+\tau}^{(1)}-d_{n}^{(1)}\right)(t-n)+\frac{1}{2 !}\left(d_{n+\tau}^{(2)}-d_{n}^{(2)}\right)(t-n)^{2}+\cdots \\
& \quad+\frac{1}{(N-1) !}\left(d_{n+\tau}^{(N-1)}-d_{n}^{(N-1)}\right)(t-n)^{N-1}+\frac{q}{N !}\left(c_{n+\tau}-c_{n}\right)(t-n)^{N} \\
& \quad+\int_{n+\tau}^{t+\tau} \int_{n+\tau}^{t_{N}} \cdots \int_{n+\tau}^{t_{2}} f\left(t_{1}\right) d t_{1} d t_{2} \cdots d t_{N}-\int_{n}^{t} \int_{n}^{t_{N}} \cdots \int_{n}^{t_{2}} f\left(t_{1}\right) d t_{1} d t_{2} \cdots d t_{N} \mid \\
& \leq \quad\left(1+|p|+\frac{|q|}{N !}+\sum_{i=0}^{N-1} \frac{1}{i !}\right) \varepsilon
\end{aligned}
$$

It follows from definition that $w(t)$ is almost periodic.

Theorem 2.11. Suppose that $|p| \neq 1$ and all eigenvalues of $A$ in (2.14) are simple (denoted by $\left.\lambda_{1}, \lambda_{2}, \ldots, \lambda_{N+1}\right)$ and satisfy $\left|\lambda_{i}\right| \neq 1,1 \leq i \leq N+1$. Then (1.1) has a unique almost periodic solution $\bar{x}(t)$, which can, in fact be determined explicitly in terms of $w(t)$ as defined in the proof of Lemma 2.8 . 
Proof. Consider the following.

Case $1(|p|<1)$. For each $m \in \mathbb{Z}^{+}$define $x_{m}(t)$ as follows:

$$
\begin{gathered}
x_{m}(t)=w(t)-p x_{m}(t-1), \quad t>-m, \\
x_{m}(t)=\phi(t), \quad t \leq-m,
\end{gathered}
$$

here $w(t)$ is as defined in the proof of Lemma 2.8, and

$$
\phi(t)=c_{n}+\left(c_{n+1}-c_{n}\right)(t-n), \quad n \leq t<n+1, n \in \mathbb{Z},
$$

where $c_{n}$ is the first component of the solution $v_{n}$ of (2.14) given by Lemma 2.9. Let $l \in \mathbb{Z}^{+}$, then from (2.21) we get

$$
(-p)^{l} x_{m}(t-l)=(-p)^{l} w(t-l)+(-p)^{l+1} x_{m}(t-l-1), \quad t>-m
$$

It follows that

$$
x_{m}(t)=\sum_{j=0}^{l-1}(-p)^{j} w(t-j)+(-p)^{l} x_{m}(t-l), \quad t>-m .
$$

If $l>t+m, x_{m}(t-l)=\phi(t-l)$, and so for such $l$,

$$
\left|x_{m}(t)-\sum_{j=0}^{l-1}(-p)^{j} w(t-j)\right| \leq|p|^{l}|\phi(t-l)| .
$$

Let $l \rightarrow \infty$, we get

$$
x_{m}(t)= \begin{cases}\sum_{j=0}^{\infty}(-p)^{j} w(t-j), & t>-m, \\ \phi(t), & t \leq-m .\end{cases}
$$

Since $w(t)$ and $\phi(t)$ are uniformly continuous on $\mathbb{R}$, it follows that $\left\{x_{m}(t): m \in \mathbb{Z}^{+}\right\}$ is equicontinuous on each interval $[-L, L], L \in \mathbb{Z}^{+}$, and by the Ascoli-Arzelá Theorem, there exists a subsequence, which we again denote by $x_{m}(t)$, and a function $\bar{x}(t)$ such that $x_{m}(t) \rightarrow \bar{x}(t)$ uniformly on $[-L, L]$, and by a familiar diagonalization procedure, can find a subsequence, again denoted by $x_{m}(t)$ which is such that $x_{m}(t) \rightarrow \bar{x}(t)$ for each $t \in \mathbb{R}$. From (2.27) it follows that

$$
x_{m}(t)=\sum_{j=0}^{\infty}(-p)^{j} w(t-j)
$$


and so $\bar{x}(t)$ is almost periodic since $w(t-j)$ is almost periodic in $t$ for each $j \geq 0$, and $|p|<1$. From (2.21), letting $m \rightarrow \infty$, we get $\bar{x}(t)+p \bar{x}(t-1)=w(t), t \in \mathbb{R}$, and since $w(t)$ solves (1.1), $\bar{x}(t)$ does also. The uniqueness of $\bar{x}(t)$ as an almost periodic solution of (1.1) follows from the uniqueness of the almost periodic solution $v_{n}: \mathbb{Z} \rightarrow \mathbb{R}^{N+1}$ of (2.14) given by Lemma 2.9, which determines the uniqueness of $w(t)$, and therefore from (2.21) the uniqueness of $\bar{x}(t)$.

Case $2(|p|>1)$. Rewriting $(2.24)$ as

$$
\left(\frac{-1}{p}\right)^{l} x_{m}(t-l)=\left(\frac{-1}{p}\right)^{l} w(t-l)+\left(\frac{-1}{p}\right)^{l+1} x_{m}(t-l-1), \quad t>-m,
$$

we deduce in a similar manner that

$$
x_{m}(t)= \begin{cases}\sum_{j=0}^{\infty}\left(\frac{-1}{p}\right)^{j} w(t-j), & t>-m \\ \phi(t), & t \leq-m .\end{cases}
$$

The remainder of the proof is similar to that of Case 1, we omit the details.

If $p=0$, the system of difference equations (2.10) of Lemma 2.7 now becomes

$$
\begin{aligned}
& c_{n+1}=\left(1+\frac{1}{N !} q\right) c_{n}+d_{n}^{(1)}+\frac{1}{2 !} d_{n}^{(2)}+\cdots+\frac{1}{(N-1) !} d_{n}^{(N-1)}+f_{n}^{(1)}, \\
& d_{n+1}^{(1)}=\frac{1}{(N-1) !} q c_{n}+d_{n}^{(1)}+d_{n}^{(2)}+\frac{1}{2 !} d_{n}^{(3)}+\cdots+\frac{1}{(N-2) !} d_{n}^{(N-1)}+f_{n}^{(2)}, \\
& \quad \vdots \\
& d_{n+1}^{(N-2)}=\frac{q}{2} c_{n}+d_{n}^{(N-1)}+d_{n}^{(N-2)}+f_{n}^{(N-1)}, \\
& d_{n+1}^{(N-1)}=q c_{n}+d_{n}^{(N-1)}+f_{n}^{(N)},
\end{aligned}
$$

and system (2.14) reduces to

$$
v_{n+1}^{*}=A^{*} v_{n}^{*}+h_{n}^{*}
$$

where

$$
A^{*}=\left(\begin{array}{cccccc}
1+\frac{q}{N !} & 1 & \frac{1}{2 !} & \cdots & \frac{1}{(N-2) !} & \frac{1}{(N-1) !} \\
\frac{q}{(N-1) !} & 1 & 1 & \cdots & \frac{1}{(N-3) !} & \frac{1}{(N-2) !} \\
\frac{q}{(N-2) !} & 0 & 1 & \cdots & \frac{1}{(N-4) !} & \frac{1}{(N-3) !} \\
\cdot & \cdot & \cdot & \cdot & \cdot & \cdot \\
\frac{q}{2 !} & 0 & 0 & \cdots & 1 & 1 \\
q & 0 & 0 & \cdots & 0 & 1
\end{array}\right)
$$


and $v_{n}^{*}=\left(x(n), y_{1}(n), y_{2}(n), \ldots, y_{N-1}\right)^{T}, h_{n}^{*}=\left(f_{n}^{(1)}, f_{n}^{(2)}, \ldots, f_{n}^{(N)}\right)^{T}$. Then we have the following theorem.

Theorem 2.12. Let $p=0$ and $q \neq(-1)^{N} N$ !, if all eigenvalues of $A^{*}$ in (2.32) are simple (denoted by $\left.\lambda_{1}, \lambda_{2}, \ldots, \lambda_{N}\right)$ and satisfy $\left|\lambda_{i}\right| \neq 1,1 \leq i \leq N$, then (1.1) has a unique almost periodic solution $\bar{x}(t)$.

Proof. System (2.32) has a solution on $\mathbb{Z}$ since $A^{*}$ is nonsingular because $q \neq(-1)^{N} N$ !. The rest of the proof follows in the same way as the proof of Theorem 2.11 and is omitted.

\section{Funding}

This paper was supported by NNSF of China and NSF of Guangdong Province (1015160150100003).

\section{References}

[1] R. Yuan, "The existence of almost periodic solutions to two-dimensional neutral differential equations with piecewise constant argument," Scientia Sinica A, vol. 27, no. 10, pp. 873-881, 1997.

[2] Z. Li and M. He, "The existence of almost periodic solutions of second order neutral differential equations with piecewise constant argument," Northeastern Mathematical Journal, vol. 15, no. 3, pp. 369-378, 1999.

[3] G. Seifert, "Second-order neutral delay-differential equations with piecewise constant time dependence," Journal of Mathematical Analysis and Applications, vol. 281, no. 1, pp. 1-9, 2003.

[4] R. Yuan, "A new almost periodic type of solutions of second order neutral delay differential equations with piecewise constant argument," Science in China. Series A, vol. 43, no. 4, pp. 371-383, 2000.

[5] R. Yuan, "Pseudo-almost periodic solutions of second-order neutral delay differential equations with piecewise constant argument," Nonlinear Analysis. Theory, Methods \& Applications, vol. 41, pp. 871-890, 2000.

[6] K. L. Cooke and J. Wiener, "A survey of differential equations with piecewise continuous arguments," in Delay Differential Equations and Dynamical Systems, vol. 1475, pp. 1-15, Springer, Berlin, Germany, 1991.

[7] A. M. Fink, Almost Periodic Differential Equations, vol. 377 of Lecture Notes in Mathematics, Springer, Berlin, Germany, 1974. 


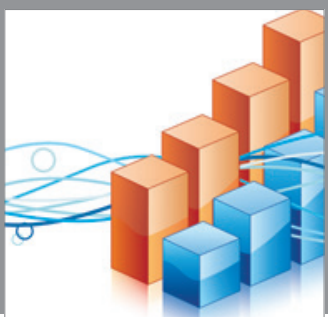

Advances in

Operations Research

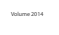

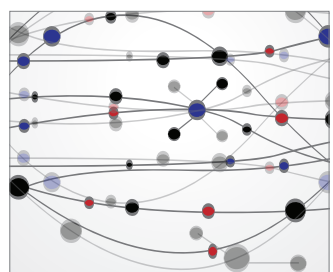

\section{The Scientific} World Journal
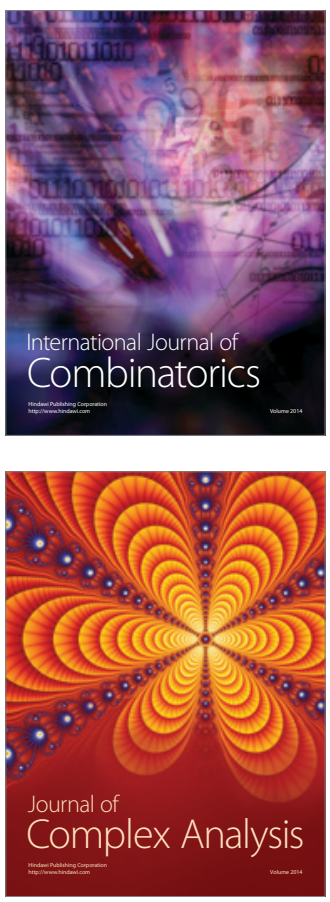

International Journal of

Mathematics and

Mathematical

Sciences
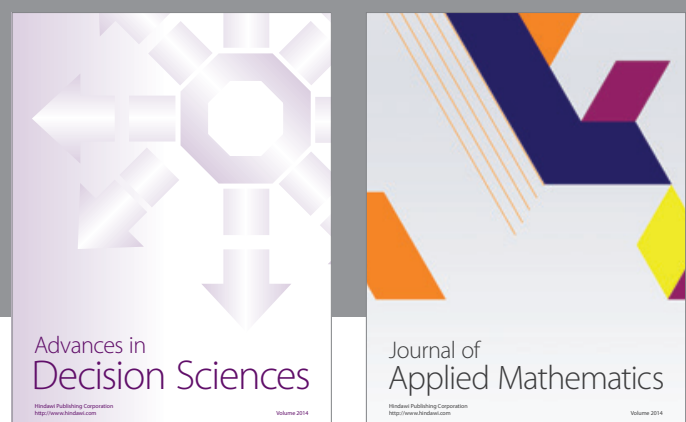

Journal of

Applied Mathematics
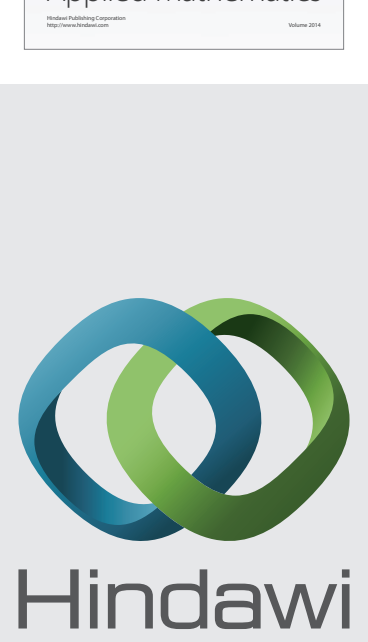

Submit your manuscripts at http://www.hindawi.com
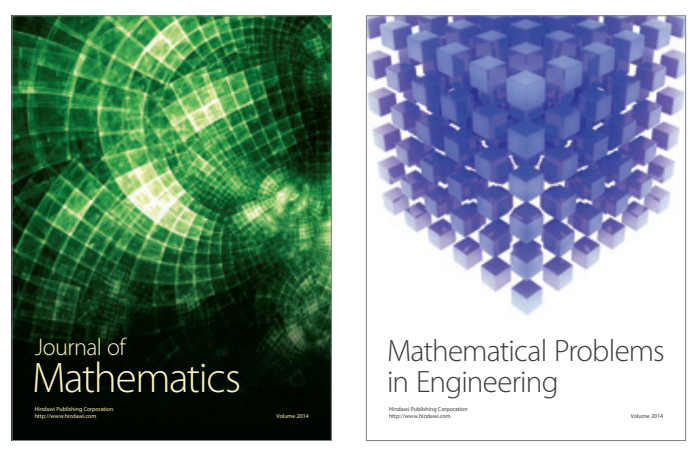

Mathematical Problems in Engineering
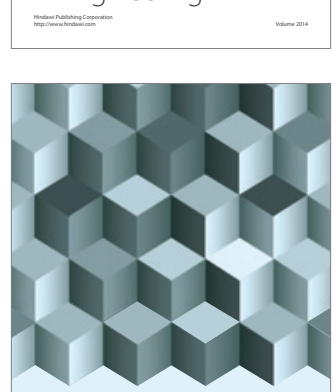

Journal of

Function Spaces
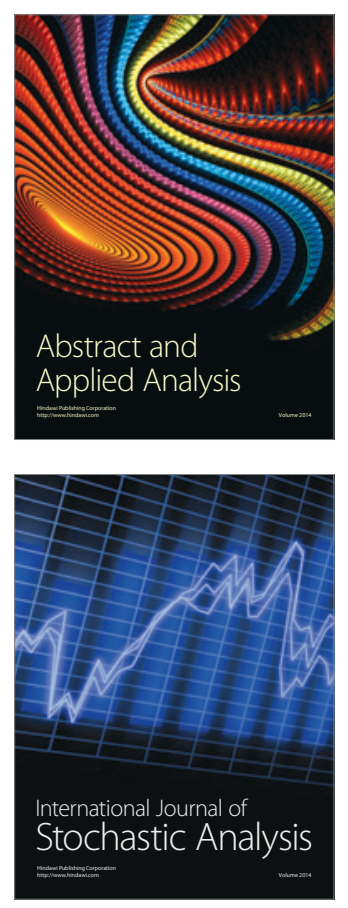

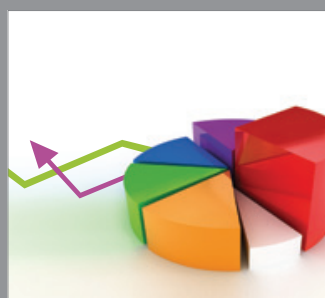

ournal of

Probability and Statistics

Promensencen
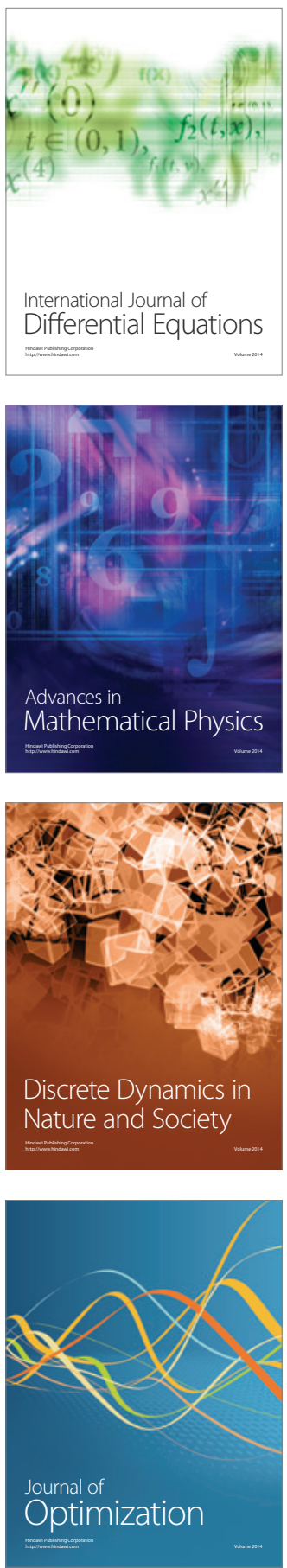